\title{
Birth and Growth of Neuroimaging and Vascular Intervention at Sree Chitra Tirunal Institute for Medical Sciences \& Technology, Thiruvananthapuram-Part II
}

\author{
Rajanikanth Rao Vedula1,2,๑ Ravi Mandalam Kolathu²,3,๑ \\ ${ }^{1}$ Krishna Institute of Medical Sciences, Secunderabad, Telangana \\ State, India \\ 2Sree Chitra Tirunal Institute for Medical Sciences and Technology, \\ Thiruvananthapuram, India \\ ${ }^{3}$ KPJ Sabah Specialist Hospital, Jalan Bersatu, Luyang, Kota Kinabalu, \\ Malaysia \\ J Clin Interv Radiol ISVIR 2021;6:37-42. \\ Address for correspondence Rajanikanth Rao Vedula, MD, DMRD, \\ FRCP (Glasgow), Apt \# 101, Woods, Somajiguda, \\ Rajbhavan Road, Hyderabad 500082, India \\ (e-mail:vedula@gmail.com).
}

\begin{abstract}
Keywords

- vascular radiology

- interventional neuroradiology

- neuroimaging

This is the second part of the two-part paper on the history of development of neuroradiology at the Sree Chitra Tirunal Institute of Medical Sciences and Technology, Thiruvananthapuram. In this part, the authors describe the evolution of interventional procedures at their institute from the initial techniques of free-flow embolization as a palliative procedure for large arteriovenous malformations (AVMs) to the more refined techniques of selective embolization using microcatheters, calibrated leak balloons and liquid polymerizing embolic agents. The authors acknowledge the immense encouragement and support provided to them by the French neurointervention pioneers Prof. Luc Picard and Prof. Jacques Moret in this endeavor.
\end{abstract}

Remember to celebrate milestones as you prepare for the road ahead.......Nelson Mandela

\section{Interventional Neuroradiology}

With the establishment of routine catheter diagnostic cerebral angiography, an increasing number of neurovascular lesions such as vascular occlusions, ectasia, displacement of vessels by space-occupying lesions (SOLs), Moyamoya disease, aneurysms, arteriovenous malformations (AVMs), arteriovenous fistula (AVF), etc., were being detected and demonstrated. In those early years, angiography was the mainstay of diagnosis for SOLs in brain in the absence of CT scan. The myth of rarity of cerebral aneurysms in Indian population was dispelled by cerebral angiography of almost every patient with suspected subarachnoid hemorrhage (SAH). Angiography was performed on strong clinical

published online April 14, 2021
DOI https://doi.org/ $10.1055 / \mathrm{s}-0041-1726145$ ISSN 2457-0214. suspicion irrespective of the findings in lumbar puncture, resulting in a significant number of cerebral aneurysms coming to light and successfully operated. The institute gained popularity for the surgery of cerebral aneurysms by Prof. George Mathews and Prof. Damodar Rout. Patients were referred from far and wide for aneurysm treatment. Incidence of cerebral AVM was much less compared to aneurysms. Serial angiography using a $70 \mathrm{~mm}$ camera at a film transit speed of 3 per second was adequate to look for the hemodynamics of AVMs. There was adequate time to examine the serial images in detail on the Heliophos projector during neuroradiology meetings every Saturday morning along with the neurologists and neurosurgeons. Cases of the week were discussed, and management was planned in these morning meetings, which helped the radiologists be clinical radiologists, rather than being just technical, an image respected by the clinical colleagues and cherished by the radiologists in the department.

(c) 2021. Indian Society of Vascular and Interventional Radiology. This is an open access article published by Thieme under the terms of the Creative Commons Attribution-NonDerivative-NonCommercial-License, permitting copying and reproduction so long as the original work is given appropriate credit. Contents may not be used for commercial purposes, or adapted, remixed, transformed or built upon. (https://creativecommons.org/licenses/by-nc-nd/4.0/). Thieme Medical and Scientific Publishers Pvt. Ltd. A-12, 2nd Floor, Sector 2, Noida-201301 UP, India 


\section{Particulate Embolization (Muscle Fibers, Development of Silastic Beads, Lyodura, Hydrogel)}

Flow-related phenomena in AVMs such as steal, collateral flow, ectasia, laminar flow patterns, etc., were recognized with great curiosity on the serial images obtained at a higher speed. Small- and medium-sized AVMs were operated by the neurosurgeons but the larger ones were mostly on follow-up if eloquent areas were involved or indication to operate was not clear. Often the indication to operate versus conservative observation in each given patient was debated in the neuroradiology meetings. This was the time published articles on endovascular treatment of brain AVMs by the French and American authors in reputed international journals such as Journal of Neuroradiology, American Journal of Neuroradiology, American Journal of Radiology, to name a few, attracted the attention of the authors. Dr. George Mathew endorsed our view that we should attempt to treat the AVMs by the interventional radiology technique. The procedure was a nonselective and nontargeted embolization using particulate material using routine fluoroscopy. VRK would dissect a small piece of muscle, 1 to $2 \mathrm{~cm}$ size, from the patient's own quadriceps muscle under local anesthesia just before the procedure. This piece of muscle was teased into smaller fibers, mixed with contrast medium, and delivered into the internal carotid artery through a $7 \mathrm{~F}$ or $8 \mathrm{~F}$ Bernstein/Judkins/Newton catheter under continuous fluoroscopic observation. It was the flow and rapidity of circulation that was monitored with extreme vigilance while talking to the patient to ensure sensorium of the patient. The patient was asked to squeeze a soft ball intermittently to sense any sensory motor weakness during the procedure. As mentioned earlier, the patient's head and neck were turned on the table for the lateral fluoroscopy. Noncooperative patients were restrained using Velcro straps. With the progress of delivery of the embolic muscle fragments and slowing of the arteriovenous transit speed, the end point of the embolization procedure was determined to check if any stasis or reflux or marked slowing were observed on fluoroscopy. A 45-year-old female patient, having a large AVM in posterior fossa near brainstem, was referred by the late Prof. Sambasivan, then at Trivandrum Medical College, for embolization. As it turned out to be an AVF, a single bolus of muscle pieces injected into the vertebral artery through a 7F Bernstein catheter completely eliminated the shunt by occluding the fistulous communication, and circulation was restored to normal. This successful procedure increased the confidence of neurosurgeons in endovascular procedures. In some patients, repeat embolizations over a period of 3 to 6 months were performed depending on the residual size of the AVM. Subsequently, conscious sedation by fentanyl and droperidol combination was used by the anesthetists for a comfortable procedure for both the patient and the radiologist. It was noted in the literature that lyophilized dura mater was yet another promising embolic particulate agent and nondegradable. The department of pathology had many specimens of brain in formaldehyde, providing us an excellent opportunity to handle the $10 \mathrm{~mm}$ slices of brain in coronal and sagittal for detailed examination of anatomy and pathology. We are fortunate to have studied the coronal and sagittal autopsy specimen slices, including the posterior fossa contents, in the early 80s itself, comparable to the present-day MRI or CT scan reformatted sections. From the knowledge of literature, the dura mater of the brain specimen was fixed in glutaraldehyde for 4 weeks instead of formaldehyde in order to eliminate its antigenicity. Arteries and veins adherent to the dura were carefully peeled out, since the vessel walls could harbor antigens. After washing the dura mater thoroughly for nearly 45 minutes in tap water, the dried dura was lyophilized in liquid nitrogen available in the pathology department. The material was cut into $10 \mathrm{~mm}$ pieces and further tiny particulate dura mater was obtained in a cryotome. The dural particles were packed in small polyethylene pouches and gas sterilized for clinical use. It was extremely gratifying to have sent the "LYODURA," as it was named after a person no less than Prof. B. Ramamurthy, doyen of neurosurgeons of India, on his personal request to the senior author. It was a letter to cherish.

Realizing that a nondegradable biocompatible particle would be ideal for delayed nonrecanalization of the AVM following embolization, silastic microspheres were considered more suitable. The biomedical wing of the institute quickly developed the product and tested for recommended toxicology and biocompatibility tests under the guidelines of the institute. On approval of the ethics committee, the microspheres were used in patients as graded particulate emboli, but with limitation of its floatation property in saline and nonadherent to contrast medium with poor visibility. Prof. A. Jayakrishnan, the Head of Polymer Chemistry Division, now Vice Chancellor of Kerala University, innovatively synthesized hydrophilic microspheres with extraordinary properties. The spheres were extruded into micron sizes (150-1000 $\mu$ ) by grade filtration. The microspheres absorb fluid and swell in size. They are compressible and deliverable through even microcatheters of 3F size distally into the feeding arteries. The microspheres were delivered into a canine renal artery, and 6 months later, the kidney was markedly shrunken on gross examination. Histopathological study did not reveal any inflammatory or giant cell reaction (-Fig. 1). Intact microspheres without any fragmentation or deformation were demonstrated like globular vacuoles on microscopy. On a limited clinical trial with approval, selective embolization of anterior spinal artery for an intramedullary cervical AVM

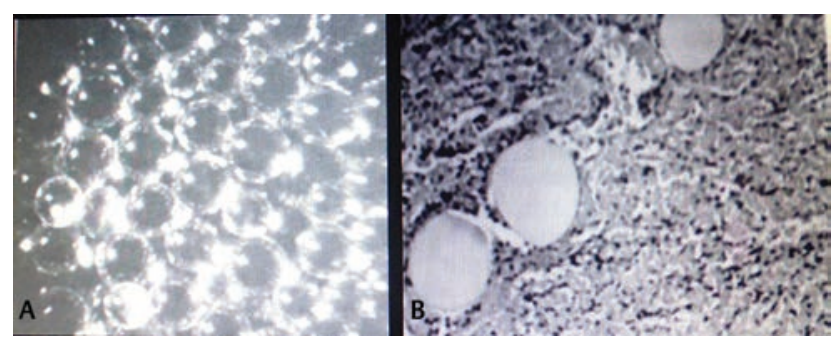

Fig. 1 (A, B) Hydrogel microspheres. (A) Gross view of the graded microspheres 250 microns in size are shown. (B) Photomicrograph shows intact microspheres in the lumen of vessels without any inflammatory reaction. 
and a large tentorial meningioma were performed with complete devascularization by obliterating the feeding arteries. Meningioma was subsequently operated with much reduced blood loss and operative time ( - Fig. 7 A, B). Significant symptomatic relief was noted in the young patient having an intramedullary cervical spinal cord AVM. Publication of this experience with Hydrogel microspheres earned us recognition for our efforts. ${ }^{1,2}$

\section{Lucien Picard and Jack Moret}

The satellite conference of the World Congress of Neurosurgery was hosted at Sree Chitra Tirunal Institute in 1984. The Department of Radiology had the privilege to interact with the giants in neurosciences, Theodore Rasmussen along with Profs. Wadia, Ramamurty, P.N. Tandon, Kak, and several other reputed neuroscientists for 3 days at Trivandrum, from where all of us travelled to Madurai for the National Conference of Neurosurgery. It was a great event to have Prof. Rasmussen inaugurate the single slice whole body Hitachi CT scanner, the first whole body scanner in the southern part of India. Drs. Picard and Moret spent a great deal of time with us in the Radiology Department, discussing the techniques of endovascular treatment of AVMs (-Fig. 2). The mentors performed partial embolization of a large AVM using the material they had brought from Paris. While leaving for the airport, Dr. Picard left an assembly of the catheter delivery system for us to study later, a photograph of which is well preserved. The main components of the assembly were the injection chamber, calibrated leak balloon, tungsten microgranules, liquid monomer 2-Isobutyl cyanoacrylate (IBCA), and silastic catheter (1.5 F). None of these items were available readily in the country and import would take its own time. A glass syringe model was constructed into which a 2 meter long pediatric intravenous infusion tubing (3F) was coiled, and hydrostatic pressure was generated through a side port welded with the help of a glass blower. The green colored long tubing (microcatheter) quickly uncoiled and ran out of the syringe exit. A bench model was ready at this stage. A 7F catheter was attached to the syringe to ensure smooth exit of the microcatheter. Since the tip of the microcatheter was not radiopaque, only an approximate length of the tubing from the groin to the suprasellar region was loaded into the syringe, allowing for the tortuosity of the internal carotid artery. This assembly was connected to the diagnostic angiographic catheter in the internal carotid artery, and a spurt of saline infusion generated pressure with propulsion of the tubing into the middle cerebral artery, confirmed by injection of contrast medium using a $2 \mathrm{~mL}$ syringe. This was the greatest step toward our future endeavors of superselective catheterization. The forward migration of the tubing into the intracranial arteries by means of this prototype was continued as superselective catheterization only for a few patients as a technique to standardize. Not satisfied with the injection chamber performance, a picture of the chamber brought by Prof. Picard was shown to Mr. Ramani, head of the R \& D wing of the institute. Very soon, a replica of the injection chamber with a well-fitting O-ring for air tightness, made of acrylic material, was given to us at no cost, which otherwise was very expensive in the French currency or US dollars ( - Fig. 3). The authors did not lose time in personally impressing Dr. Valiathan, the need of importing these items, and the consignment arrived in a short time as usual. It was necessary to prepare the silastic microcatheter ("Pursil" in

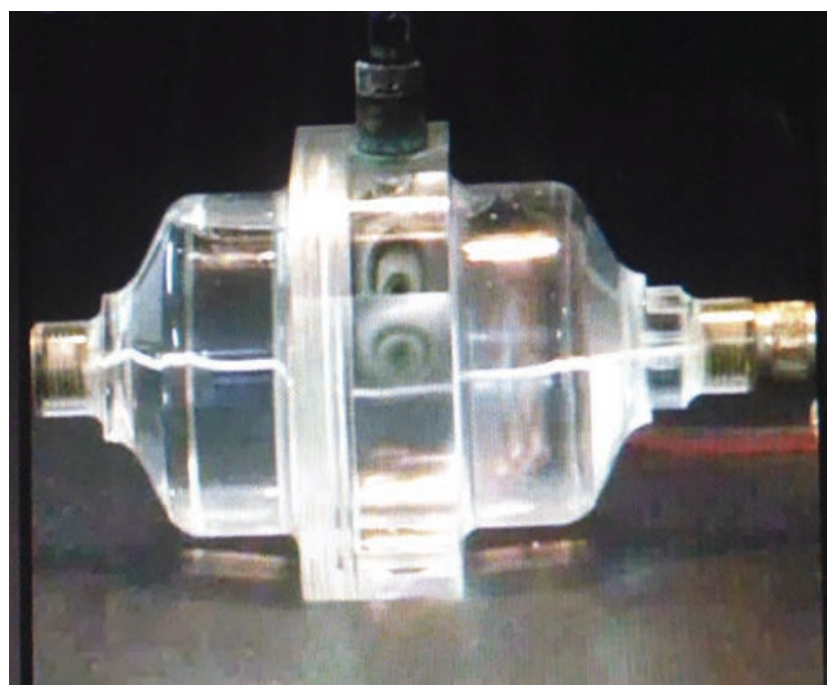

Fig. 3 Acrylic injection chamber. The propulsion chamber showing the loaded microcatheter was fabricated at the R\&D wing of the institute.

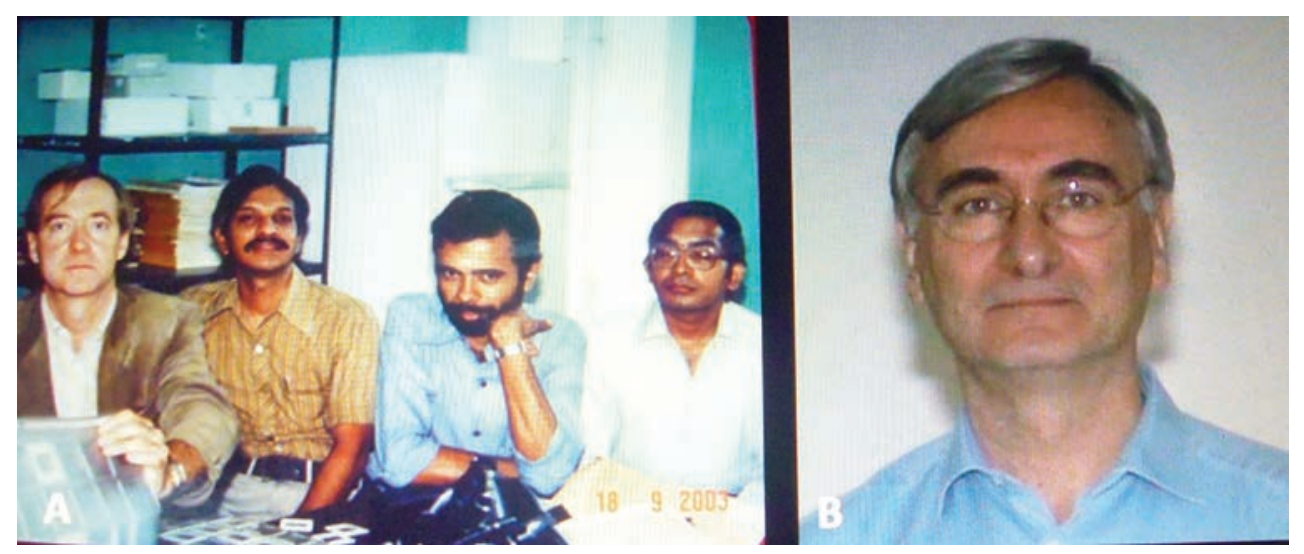

Fig. 2 (A, B) With Prof. Luc Picard and Prof. Jack Moret in 1984 during his visit to the institute. (A) From left to right: Prof. Picard, V.R.K. Rao, Ravi Mandalam, AK Gupta. (B) Prof. Jack Moret. 
100 meters roll) at both its ends, with a connecting hub and a blunt needle at one end and attachment of a gold-clip at the distal end for radio opacity during fluoroscopy. Finally, a calibrated leak balloon was prepared using a 20G needle for puncture, and it was mounted on the silastic catheter (-Fig. 4). The microcatheter was navigated as far distally as possible, and superselective angiography of branches of the middle cerebral artery, posterior cerebral artery and anterior cerebral artery were performed in a few patients to be thorough with the technique of superselective catheterization, which the French refer to as CATHETERISM! These angiographic pictures were identical to the description of vascular territories in the volume on arteries in Newton and Pott's five-volume text-Radiology of Skull and Brain. Three or four microcatheters mounted with balloons were kept ready on the day prior to the procedure. Often it would take 2 to 3 hours to reach the nidus of an AVM. The first patient for embolization was a 40-year-old female presenting with seizures having an AVM in the parietal convexity on the right side (-Fig. 5). An ideal mixture of IBCA, Myodil and tantalum powder was injected in order to penetrate the nidus of the AVM through the calibrated leak balloon and more than two thirds of the AVM bed was obliterated over two sessions. She was subsequently operated successfully with less operation time and blood transfusion. Over the coming years, more than 150 superselective catheterizations were performed and embolization was possible in a select few patients (-Fig. 6). Radioanatomic cure was feasible in more than $50 \%$ of patients, some of which were palliative. ${ }^{3-10}$ A landmark publication on dissolution of IBCA in the treated AVMs of brain in AJNR received very wide attention, with citations

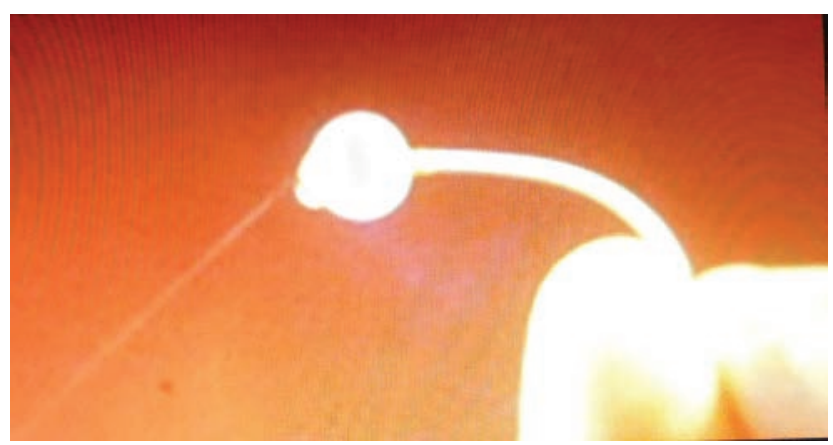

Fig. 4 Calibrated leak balloon shows the jet of saline from its tip in its inflated state, which would also prevent antegrade flow in the feeding artery. exceeding a hundred in number. ${ }^{11}$ Convinced by the quality and quantum of service for peripheral vascular disease and neurointervention, our request for two beds for interventional radiology was readily accepted by Prof. Valiathan and quickly a routine was adopted for admissions, reviews, and daily rounds of our patients appropriately involving the neurologists, neurosurgeons, and anesthetists using cross-consultations. The authors made certain that every patient to undergo any interventional procedure would be counseled thoroughly, an informed consent in the local language (Malayalam) would be read to the patient if illiterate, and signatures of patient/attender/witnesses were obtained. The consent form, adapted from the AJR and radiology literature, clearly mentions the technical procedure, benefits, risks and alternate methods of treatment in lay terminology comprehensible to the patient.

Often patients would seek information on the number of patients treated earlier by this new technique and complications including mortality for which the counseling radiologist was forthcoming with appropriate and satisfying answers. It was a standard practice to inform the neurological colleagues about the details of the scheduled procedure a day before. The neurologist would document the neurological status in the case file before the procedure to be able to assess postprocedural complications, if any, for prognosis. There was an arrangement with the department of anesthesia to have an anesthetist always during the procedure for any emergency resuscitation or intubation. Patients with any neurological deficit following intervention were immediately managed in the neurointensive care unit by the clinical colleagues. It was a learning experience to follow the rounds of clinicians and witness management of complications, often attending the surgical procedures as well. An instance of emergency craniotomy to deflate an inflated and impacted balloon in the M1 segment, a life-saving procedure exemplifies the cooperation and involvement of clinical colleagues.

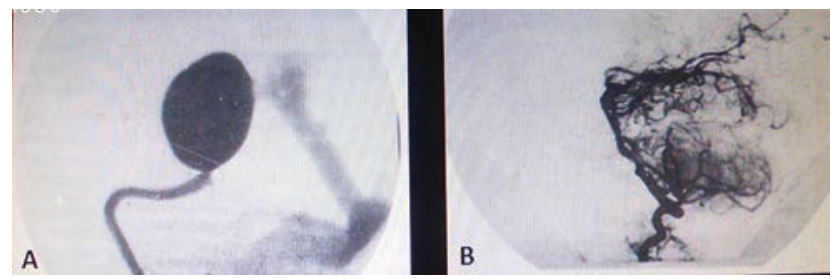

Fig. 6 (A, B) Aneurysm of vein of Galen was completely cured by injecting concentrated Isobutyl cyanoacrylate (IBCA) in two sessions.

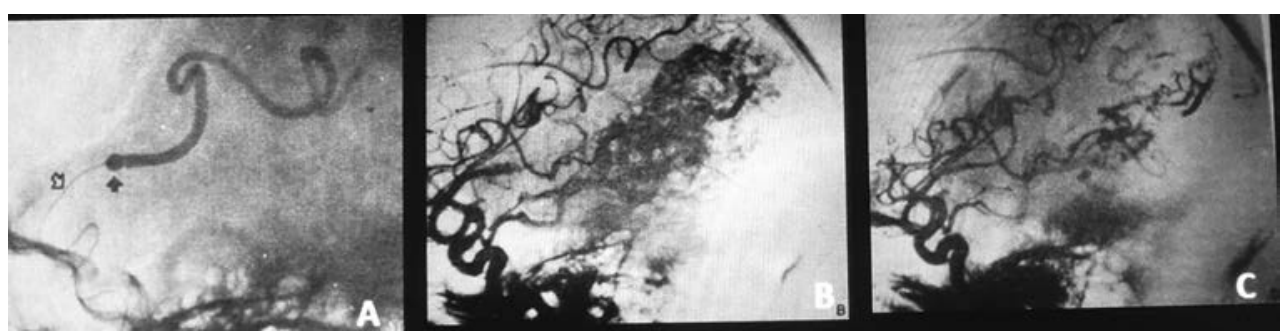

Fig. 5 (A-C). Superselective catheterization and embolization of a parietal arteriovenous malformation (AVM) using 2-Isobutyl cyanoacrylate (2-IBCA). (A) Calibrated leak balloon in the proximal middle cerebral artery progressing toward the nidus is shown. (B, C) Reduction of the size of the AVM is demonstrated in the pre $(\mathbf{B})$ and post $(\mathbf{C})$ embolization images. 


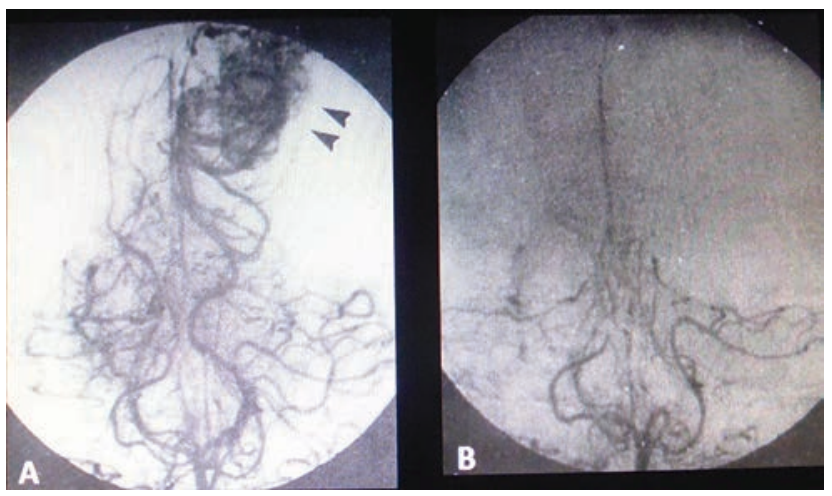

Fig. 7 (A, B) Preoperative embolization of meningioma. injection of 150 micron Hydrogel particles through a microcatheter into the parieto-occipital artery demonstrates markedly reduced vascularity of the falx meningioma.

It may be recalled that the public sector undertaking Mishra Dhatu Nigam (MIDHANI) was approached, and tantalum powder of import quality was supplied to us in bulk at no cost, thus avoiding importing. Around this time, the first patient having a carotid cavernous fistula was completely cured using detachable balloons supplied by M/s Balt Extrusion. Mr. Devang Mehta was the sole distributor of balloons, silastic thread and silastic microcatheter (1.5F) along with other interventional products. A broad range of balloons and microcatheters were procured from Balt Company through Mehta. Apart from the $2+3 \mathrm{~F}$ coaxial catheter system for detachment of balloons in the blood vessels, a double lumen microcatheter (BD-2L) was available for detachable balloon to be used with a permanent solidifying liquid agent, hydroxy ethyl methyl acrylate (HEMA).

\section{Indigenization Efforts}

Having treated around 40 carotid cavernous fistulae using contrast-filled detachable balloons, the quest for making the balloons and HEMA in India led the authors to approach $\mathrm{M} / \mathrm{s}$ Hindustan Latex Company. Scientists there were ready to make mini-balloons for our requirement if a corresponding metal/steel mould was available. The mechanical engineering group at Vikram Sarabhai Space Center (VSSC) addressed the idea of fabricating a mini-mould for the mini-balloon. Yet, the mould had its uneven corners at its tip, causing the balloon to have an uneven thickness and bursting of the inflated balloon at those weak points. Hindustan Machine Tools at Bangalore had its microtools for manufacturing watches, and it was another option to approach the organization. At the same time, the Chemicals Group of VSSC was in a position to look into the composition and properties of the liquid monomer (HEMA). Its stability, exothermic reaction, control of timing of polymerization, biodegradability, and biocompatibility were to be tested. However, preliminary synthesis of HEMA was very encouraging step, and it was filled into a balloon in vitro to be observed for leaching and material interaction, etc. Further work on these promising initiatives could not be pursued due to unavoidable reasons.

\section{Postdoctoral Training Course in Neuroradiology}

By the early 1980s, the Radiology Department at Sree Chithra Tirunal Institute had already gained a name for itself, by virtue of the range of imaging and interventional procedures that it could offer. Presentation of scientific papers at regional and national conferences and publications in peer-reviewed journals, both local and overseas, served to enhance this reputation. ${ }^{12-15}$ It was the appropriate time to start a postdoctoral training course in neuroradiology for general radiologists wishing to widen their knowledge and skills. In those days, neuroradiology formed a negligible part of radiology training curriculum in most medical colleges in India. It was also at that time, CT scan systems were sprouting all over the country, many in medical colleges and teaching institutions and some also as standalone scanning centers in the private sector. Responsibility was felt to share our knowledge and experience with young radiologists who could go on to form the core of a neuroradiology community in the country. With this intent, permission was obtained to start a postdoctoral certificate course (PDCC) of 1 year's duration. The training gave equal emphasis to theoretical knowledge as well as practical, hands-on experience on various invasive procedures. Our first trainee in 1985 was Dr. Arun Kumar Gupta, who, in subsequent years, joined our faculty and rose to become Professor and Head of Department. Several of our trainees have gone on to become accomplished interventional radiologists-Dr. Rajendra Phadke and Dr. Sunil Kumar (SGPGIMS, Lucknow), Dr. Santosh Joseph (SRMC, Chennai), Dr. Mathew Cherian (Kovai Medical Centre, Coimbatore), Dr. Swatee Halbe (Apollo Hospitals, Chennai), Dr. Sreekant Murthy (Amrita Institute of Medical Sciences, Cochin), and Dr. Jayakrishnan (Cochin) to name a few.

\section{Growth of Other Modalities}

Although the department earned its repertoire in performing a range of invasive and interventional procedures, diagnostic neuroimaging was not neglected. After the installation of the whole-body CT scanner in 1983, a full-fledged 1.5 Tesla MRI scanner system (Signa, GE Medical System) was installed in 1994 to cater to the growing need for MR brain and spine imaging. The system was inaugurated by the eminent jurist Shri Nani Palkhivala.

\section{Timeline of Events}

1976-Inauguration of Sree Chitra Tirunal Institute for Medical Sciences and Technology.

1977-Installation of Philips Diagnost 73 Fluoroscopy System.

1979-Installation of MIMER III (Elema Schonander) System.

1979-Commencement of transfemoral four-vessel cerebral angiography services. 
1980-Commencement of free-flow cerebral AVM embolization.

1983-Installation of Hitachi CT-W4 Whole Body CT scanner.

1985-Commencement of one-year Postdoctoral Certificate Course (PDCC).

1986-First superselective angiography and embolization of brain AVM.

1986-First balloon occlusion of caroticocavernous fistula.

1986-First percutaneous transluminal balloon angioplasty of subclavian artery stenosis.

1988-Installation of Siemens Bicor Digital Subtraction Angiography system.

1990-First laser angioplasty in long-segment occlusion of femoral artery.

1993-Inauguration of 1.5 Tesla Signa (GE) MRI scanner.

\section{Conflict of Interest}

None declared.

\section{Acknowledgments}

Words cannot fully justify the great support the authors had from their colleagues and students in those fruitful years at the Sree Chitra Tirunal Institute of Medical Sciences and Technology, Thiruvananthapuram. The senior author, V.R.K., has already recorded his intense appreciation, admiration and gratitude to the former Director Prof. MS Valiathan, without whose vision, dynamism and guidance, none of the achievements would have been possible. Apart from having an accomplished senior colleague and comrade in Dr. Ravi Mandalam, he was fortunate to have a very young team in the form of Dr. Arun K Gupta, Dr. Santosh Joseph, Dr. Madhavan Unni, and Dr. Srinivasa Rao. As mentioned earlier, many of the trainees, Dr. Phadke, Dr. Sunil Kumar, Dr. Swatee, Dr. Mathew Cheriyan, Dr. Srikant and Dr. Jayakrishnan, have gone on to become leading figures in the Indian interventional radiology scene and have done them proud by their achievements. I would be negligent in my duty, if I do not mention the stellar role played by my own staff-radiographers Mrs. Sherifa Beevi, Mrs. Achiamma Abraham, Mr. Hari Kumar, nursing staff Mrs. Baby Mathew, and my dark room technicians Mr. Viswanathan Nair and Mr. Reveendran, who labored long hours together with us in a common purpose and endeavor. Finally, my thanks are also due to my neurology and neurosurgical colleagues-late Prof. George Mathews, Prof. Damodar Rout, Prof. AK Bhattacharya, Prof. B.K. Misra, late Prof. PK Mohan and many others for their support and clinical inputs (R.R.V.).

\section{References}

1 Radhakrishnan VV, Saraswathy A, Rao VRK, Rout D, Jayakrishnan A. Histopathological evaluation of polymethyl methacrylate as an embolic agent. Acta Neurochir (Wien) 1992;117(1-2):30-33

2 Rao VRK, Ravimandalam K, Jayakrishnan A, et al. Hydrolysed microspheres from cross-linked polymethyl methacrylate (Hydrogel). A new embolic material for interventional neuroradiology. J Neuroradiol 1991;18(1):61-69

3 Santhosh J, Rao VRK, Ravimandalam K, Gupta AK, Unni NM, Rao AS. Endovascular management of carotid cavernous fistulae: observation on angiographic and clinical results. Acta Neurol Scand 1993;88(5):320-326

4 Rao VR, Ravimandalam K, Gupta AK, Joseph S, Unni M, Rao AS. Angiographic analysis and results of endovascular therapy of aneurysm of vein of Galen. J Neuroradiol 1994;21(3):213-222

5 Gupta AK, Rao VRK, Joseph S, et al. Vein of Galen malformations - An Indian experience. Rivista di Neuroradiologia 2003;16:1304-1307

6 Gupta AK, Rao VRK, Varma DR, et al. Evaluation, management, and long-term follow up of vein of Galen malformations. J Neurosurg 2006;105(1):26-33

7 Rao VRK, Ravimandalam K, Gupta AK, et al. Pre-embolization superselective angiography of arteriovenous malformations: technical considerations. Ind J Radiol 1987;41:357-361

8 RaoVRK, Ravimandalam K, Bhattacharya RN, etal.Percutaneous trans-catheter embolization in the management of cerebral arteriovenous malformations and cirsoid aneurysms of scalp: Preliminary experiences with isobutyl 2-cyanoacrylate. Neurol India 1988;36:81-95

9 Rao VRK, Ravimandalam K, Rout D, et al. V. Endovascular therapy of cerebral Arteriovenous malformations by superselective embolization. Ind J Radiol 1990;44:223-234

10 Kumar S, Ravimandalam K, Rao VRK, et al. Percutaneous transluminal angioplasty in supra-aortic vessels. Ind J Radiol 1990;44:239-245

11 Rao VRK, Mandalam KR, Gupta AK, Kumar S, Joseph S. Dissolution of isobutyl 2-cyanoacrylate on long-term follow-up. AJNR Am J Neuroradiol 1989;10(1):135-141

12 Rao VRK, Pillai SM, Shenoy KT, Radhakrishnan VV, Mathews G. Hypervascular cavernous angioma at angiography. Neuroradiology 1979;18(4):211-214

13 Rout D, Sharma A, Mohan PK, Rao VR. Bacterial aneurysms of the intracavernous carotid artery. J Neurosurg 1984 ;60(6):1236-1242

14 Gupta AK, Rao VRK, Mandalam KR, et al. Thrombosis of multiple aneurysms of a lateral lenticulostriate artery. An angiographic follow-up. Neuroradiology 1989;31(2):193-195

15 Kumar S, Rao VRK, Mandalam KR, Phadke RV. Disappearance of a cerebral aneurysm-an unusual angiographic event. Clin Neurol Neurosurg 1991;93(2):151-153 